\title{
DECOMPOSABILITY OF ABSTRACT AND PATH-INDUCED CONVEXITIES IN HYPERGRAPHS
}

\author{
Francesco Mario Malvestuto
}

AND

MARINA MOSCARINI

Department of Informatics

Sapienza University of Rome

Via Salaria 113, 00198 Roma, Italy

e-mail: malvestuto@di.uniroma1.it moscarini@di.uniroma1.it

\begin{abstract}
An abstract convexity space on a connected hypergraph $H$ with vertex set $V(H)$ is a family $C$ of subsets of $V(H)$ (to be called the convex sets of $H$ ) such that: (i) $C$ contains the empty set and $V(H)$, (ii) $C$ is closed under intersection, and (iii) every set in $C$ is connected in $H$. A convex set $X$ of $H$ is a minimal vertex convex separator of $H$ if there exist two vertices of $H$ that are separated by $X$ and are not separated by any convex set that is a proper subset of $X$. A nonempty subset $X$ of $V(H)$ is a cluster of $H$ if in $H$ every two vertices in $X$ are not separated by any convex set. The cluster hypergraph of $H$ is the hypergraph with vertex set $V(H)$ whose edges are the maximal clusters of $H$. A convexity space on $H$ is called decomposable if it satisfies the following three properties:

(C1) the cluster hypergraph of $H$ is acyclic,

(C2) every edge of the cluster hypergraph of $H$ is convex,

(C3) for every nonempty proper subset $X$ of $V(H)$, a vertex $v$ does not belong to the convex hull of $X$ if and only if $v$ is separated from $X$ in $H$ by a convex cluster.

It is known that the monophonic convexity (i.e., the convexity induced by the set of chordless paths) on a connected hypergraph is decomposable.

In this paper we first provide two characterizations of decomposable convexities and then, after introducing the notion of a hereditary path family in a connected hypergraph $H$, we show that the convexity space on $H$ induced
\end{abstract}


by any hereditary path family containing all chordless paths (such as the families of simple paths and of all paths) is decomposable.

Keywords: convex hull, hypergraph convexity, path-induced convexity, convex geometry.

2010 Mathematics Subject Classification: Primary: 05C65, 52A01; Secondary: 52B55.

\section{REFERENCES}

[1] C. Beeri, R. Fagin, D. Maier and M. Yannakakis, On the desirability of acyclic database schemes, J. ACM 30 (1983) 479-513. doi:10.1145/2402.322389

[2] M. Changat and J. Mathew, On triangle path convexity in graphs, Discrete Math. 206 (1999) 91-95. doi:10.1016/S0012-365X(98)00394-X

[3] M. Changat, H.M. Mulder and G. Sierksma, Convexities related to path properties on graphs, Discrete Math. 290 (2005) 117-131. doi:10.1016/j.disc.2003.07.014

[4] R. Diestel, Graph Decompositions: A Study in Infinity Graph Theory (Clarendon Press, Oxford, 1990).

[5] P. Duchet, Convexity in combinatorial structures, in: Proceedings of the 14th Winter School on Abstract Analysis, Frolik, Souček and Fabián (Eds), (Circolo Matematico di Palermo, Palermo 1987), Serie II 14 261-293

[6] P. Duchet, Convex sets in graphs II: minimal path convexity, J. Combin. Theory Ser. B 44 (1988) 307-316. doi:10.1016/0095-8956(88)90039-1

[7] P. Duchet, Discrete convexity: retractions, morphisms and the partition problem, in: Proceedings of the Conference on Graph Connections, Balakrishnan, Mulder and Vijayakumar (Ed(s)), (Allied Publishers, New Delhi, 1999) 10-18.

[8] M. Farber and R.E. Jamison, Convexity in graphs and hypergraphs, SIAM J. Algebraic Discrete Methods 7 (1986) 433-444. doi:10.1137/0607049

[9] H.-G. Leimer, Optimal decomposition by clique separators, Discrete Math. 113 (1993) 99-123. doi:10.1016/0012-365X(93)90510-Z

[10] F.M. Malvestuto, Canonical and monophonic convexities in hypergraphs, Discrete Math. 309 (2009) 4287-4298. doi:10.1016/j.disc.2009.01.003

[11] F.M. Malvestuto, Decomposable convexities in graphs and hypergraphs, ISRN Combinatorics 2013 Article ID 453808. doi:10.1155/2013/453808 
[12] F.M. Malvestuto, M. Mezzini and M. Moscarini, Equivalence between hypergraph convexities ISRN Discrete Mathematics 2011 Article ID 806193. doi:10.5402/2011/806193

[13] R.E. Tarjan, Decomposition by clique separators, Discrete Math. 55 (1985) 221-232. doi:10.1016/0012-365X(85)90051-2

[14] M. Van de Vel, Theory of Convex Structures (North-Holland Publishing Co., Amsterdam, 1993).

[15] S. Whitesides, An Algorithm for finding clique cut-sets, Inform. Process. Lett. 12 (1981) 31-32.

doi:10.1016/0020-0190(81)90072-7

Received 20 January 2014

Revised 7 October 2014

Accepted 23 October 2014 